\title{
Characterizing interactions between earthquake rupture and fault zone structure
}

Yihe Huang (University of Michigan Ann Arbor) Jean-Paul Ampuero, Don V. Helmberger (Caltech)

2016 GSA meeting

Sep 25, 2016 


\section{DFZs are detected by seismic waves}

Table 1. Summary of Material Properties of Main Fault Zones

Fault Zones

San Andreas

San Jacinto

Landers

Hector Mine

Calico

Nojima

Anatolian
Width $(\mathrm{m})$

$$
\begin{aligned}
& \sim 150 \\
& \sim 200
\end{aligned}
$$

$125-180$

$150-200$

270-360

$150-200$

$75-100$

$\sim 1500$

$\sim 1300$

100-220

$\sim 100$
Velocity Reduction (\%)

$Q_{s}$

$$
30-40
$$

$10-40$

35-45

25-60

$35-60$

$30-40$

40-50

40-50

40-50

50
20-30

$10-60$

20-40

$$
20-40
$$

10-15

[Huang et al., 2014] 


\section{How can DFZs change earthquake rupture?}

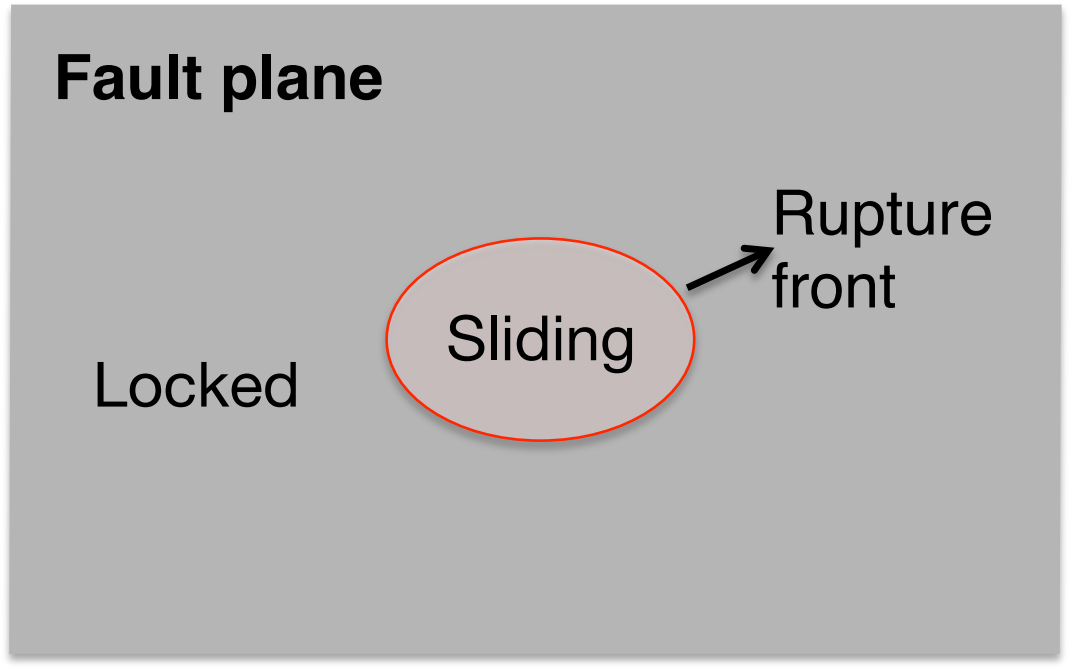




\section{How can DFZs change earthquake rupture?}

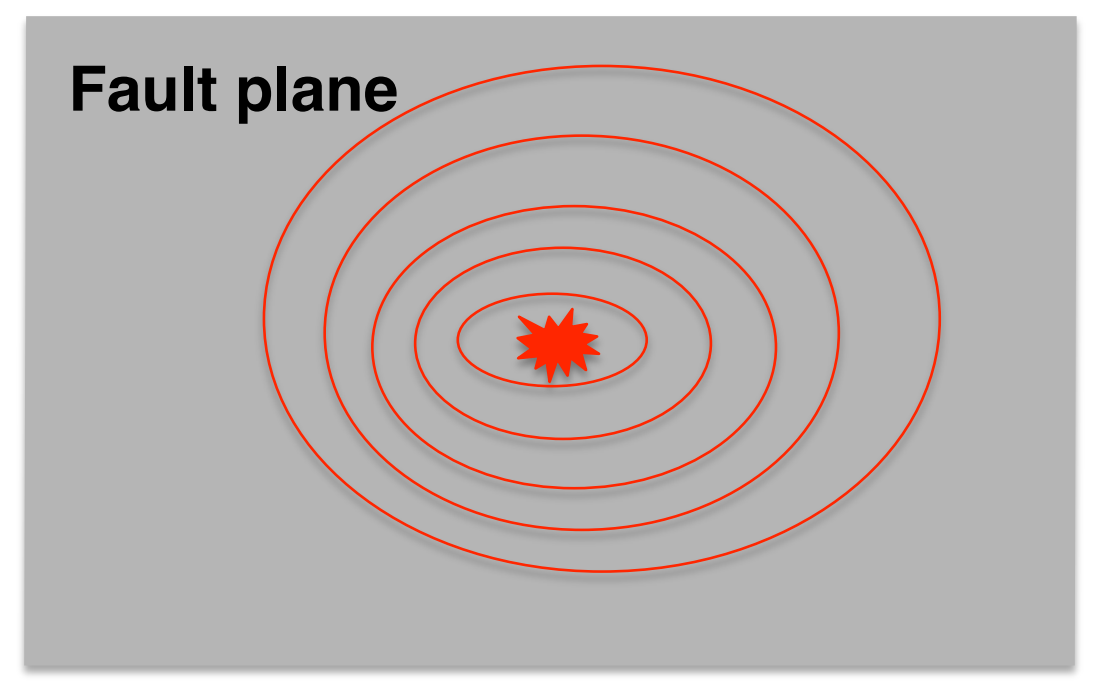

Rupture speed is how fast the rupture front propagates. 


\section{How can DFZs change earthquake rupture?}
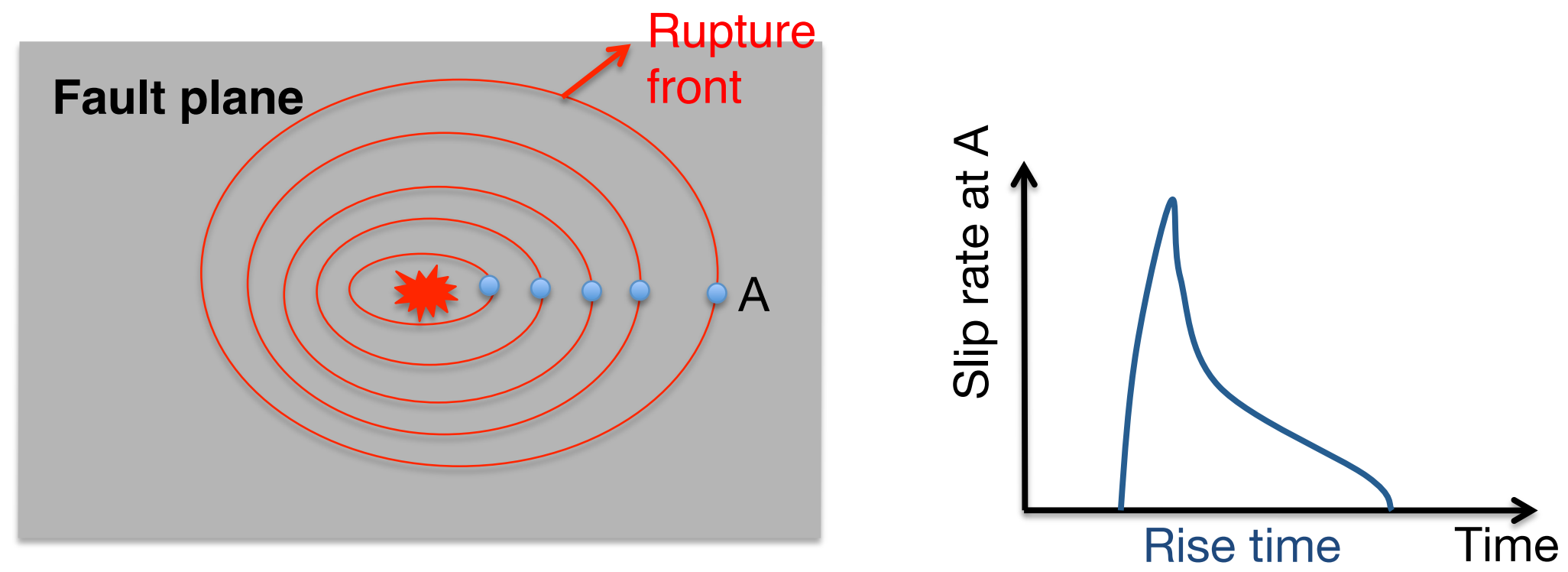

Rupture speed

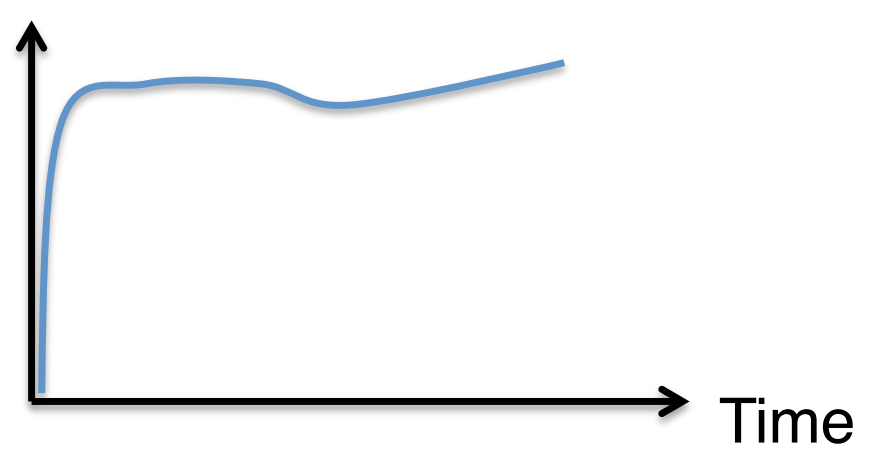




\section{DFZs trap waves and induce fault stress changes}

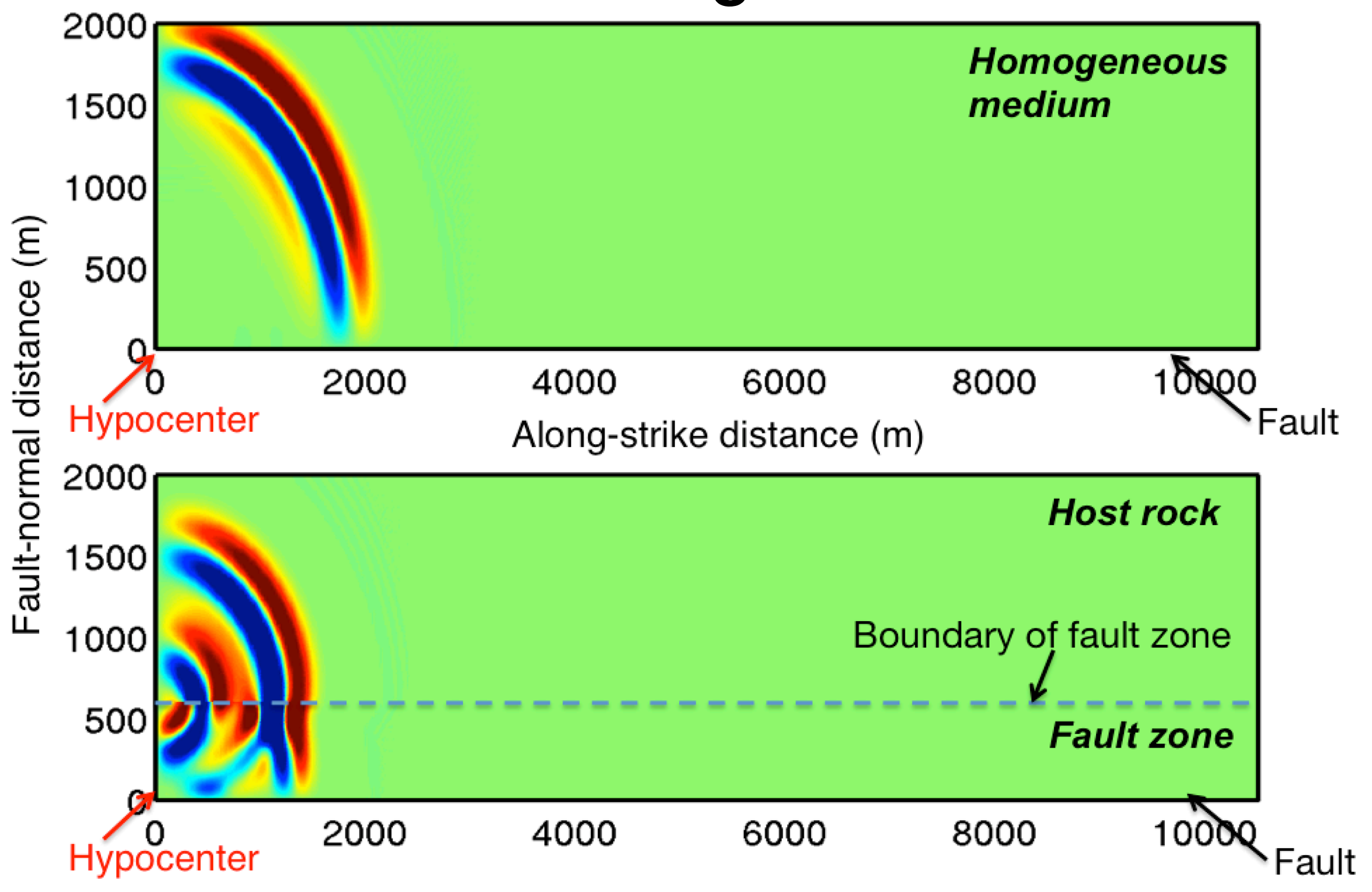




\section{DFZs trap waves and induce fault stress changes}

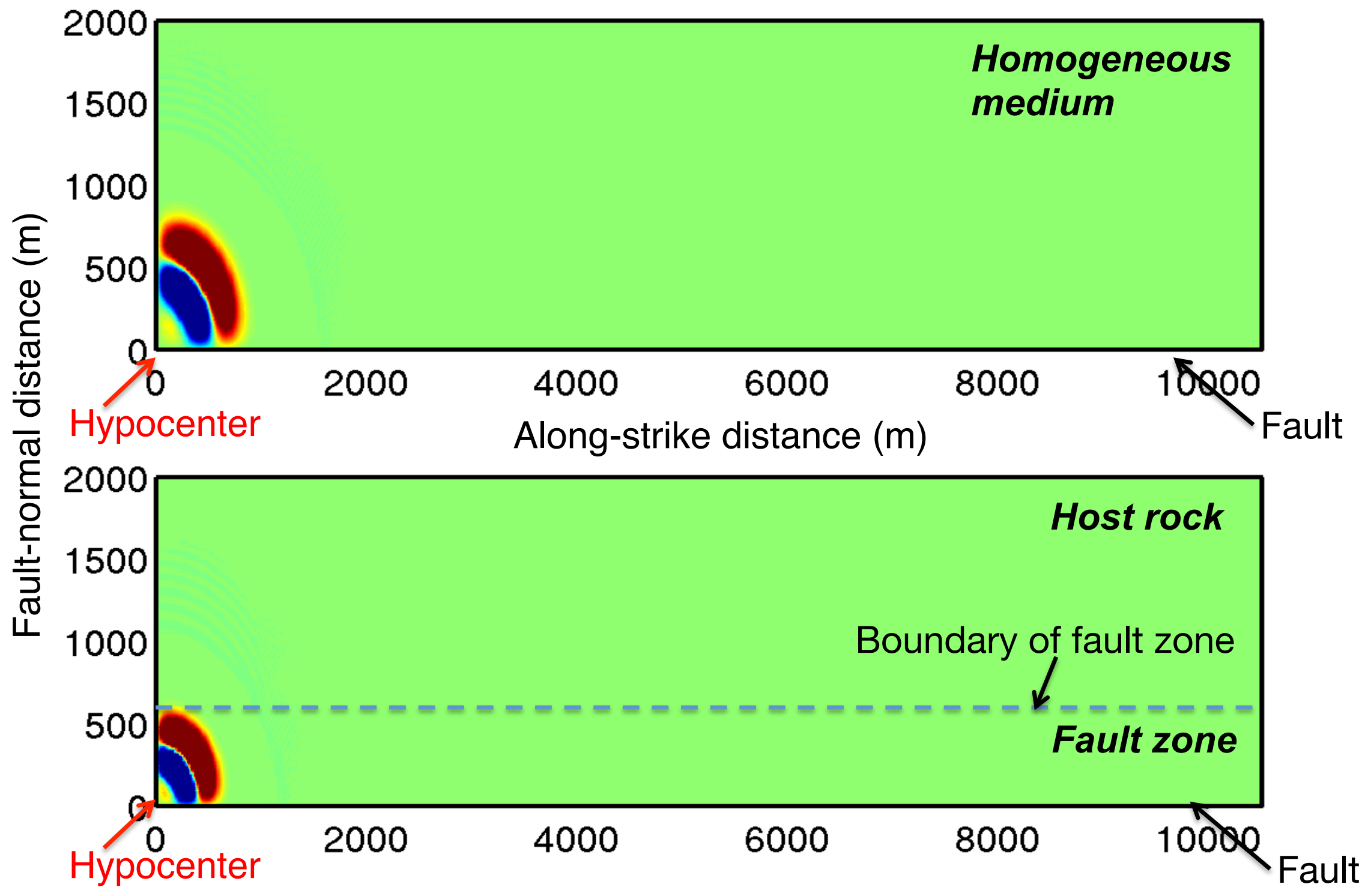




\section{DFZs trap waves and induce fault stress changes}

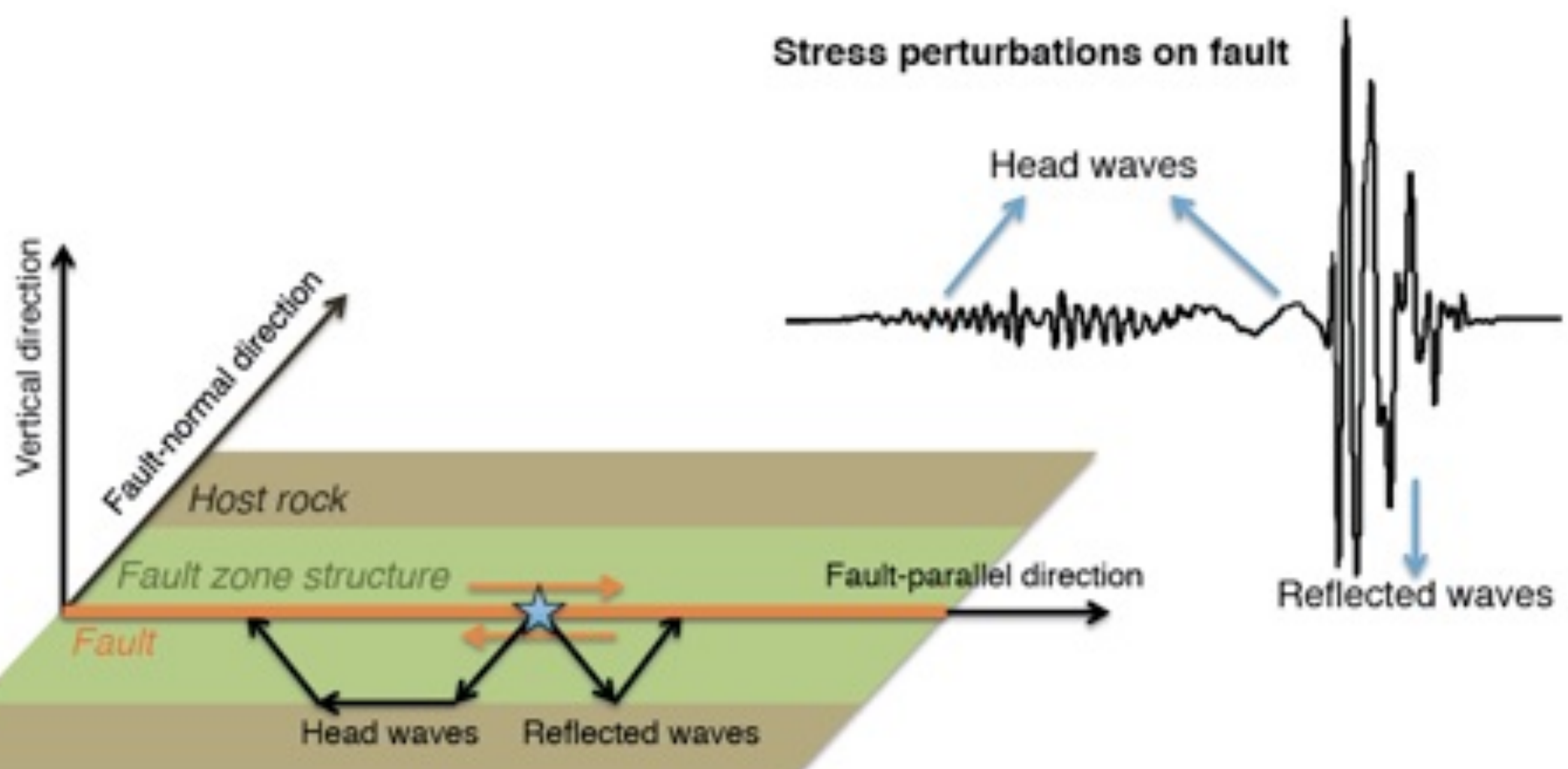




\section{Slip rate functions are altered by DFZ reflections}

Homogeneous medium

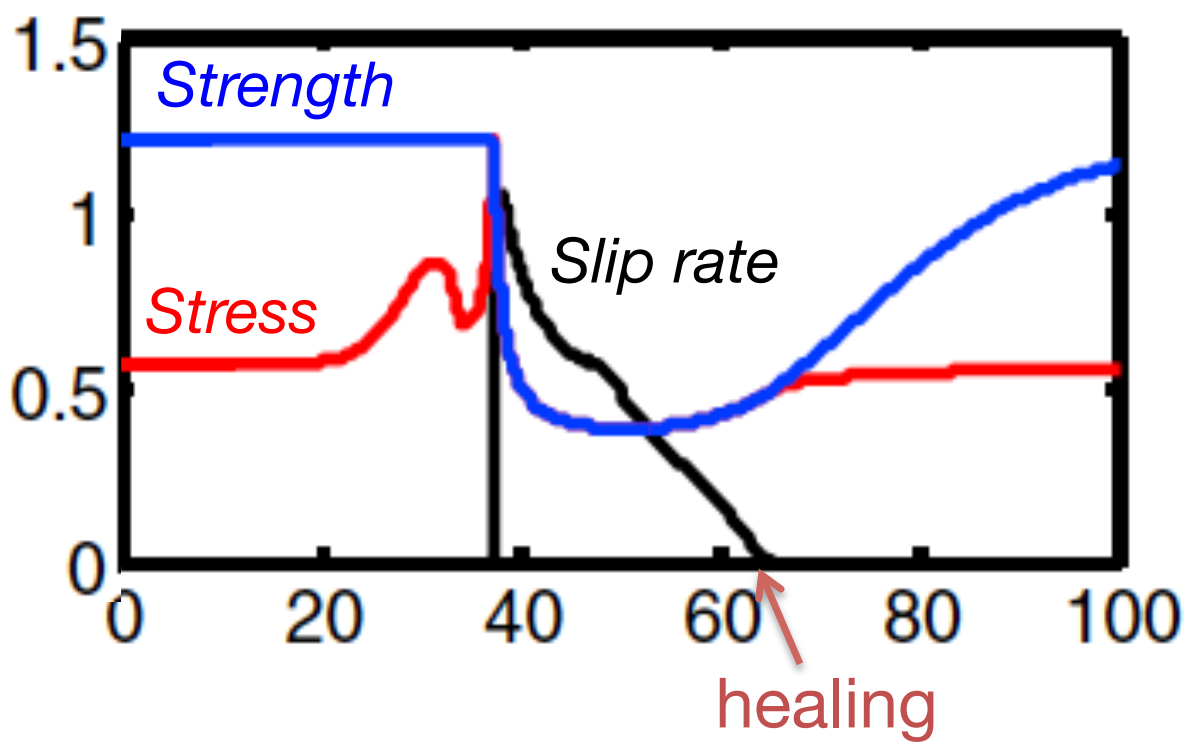

Damaged fault zones

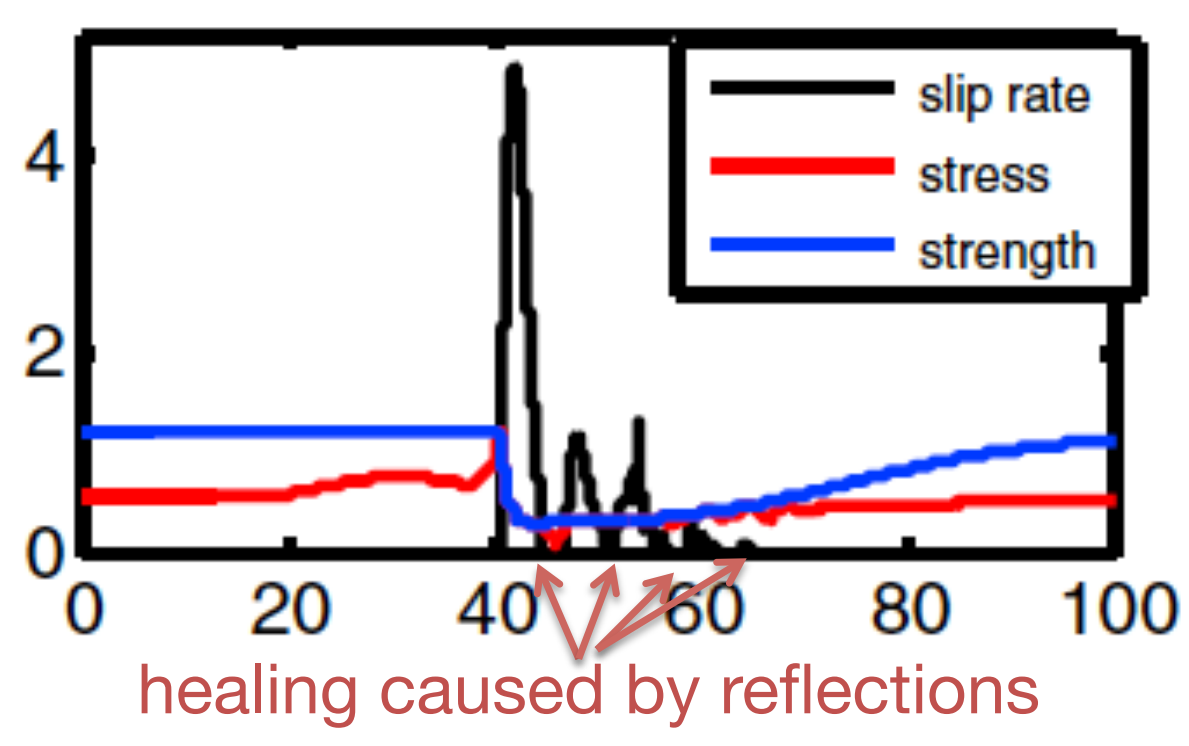

(More high-frequency ground motion!)

[Huang and Ampuero, 2011; Huang et al., 2014] 


\section{Rupture velocity is accelerated by head waves}

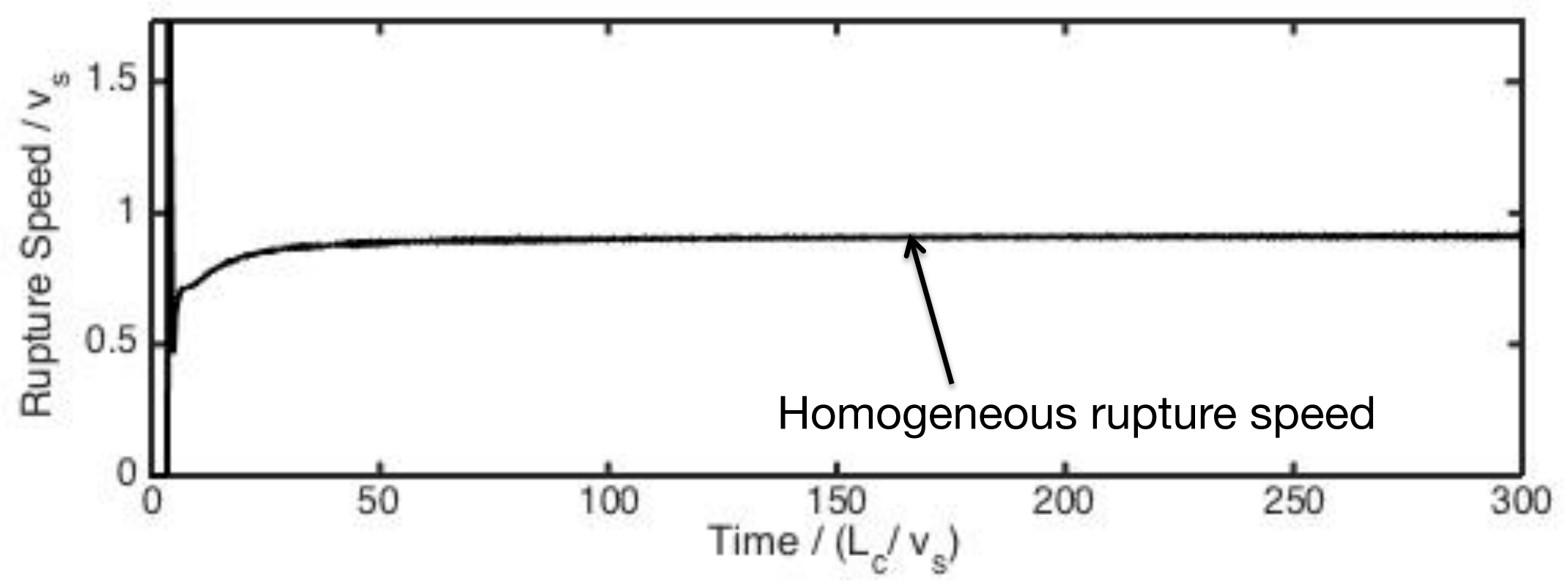




\section{Rupture velocity is accelerated by head waves}

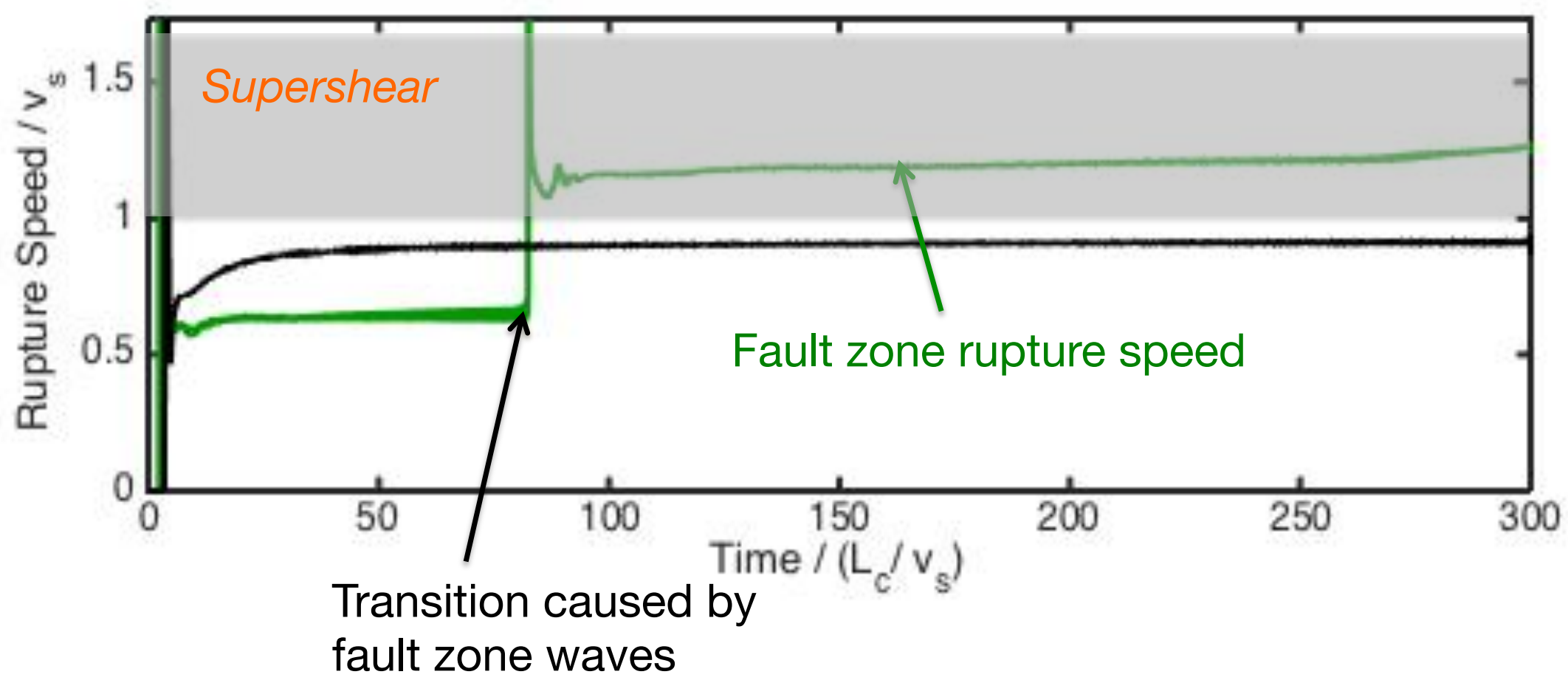

[Huang et al., 2014, 2016] 


\section{Large attenuation delays rupture acceleration}

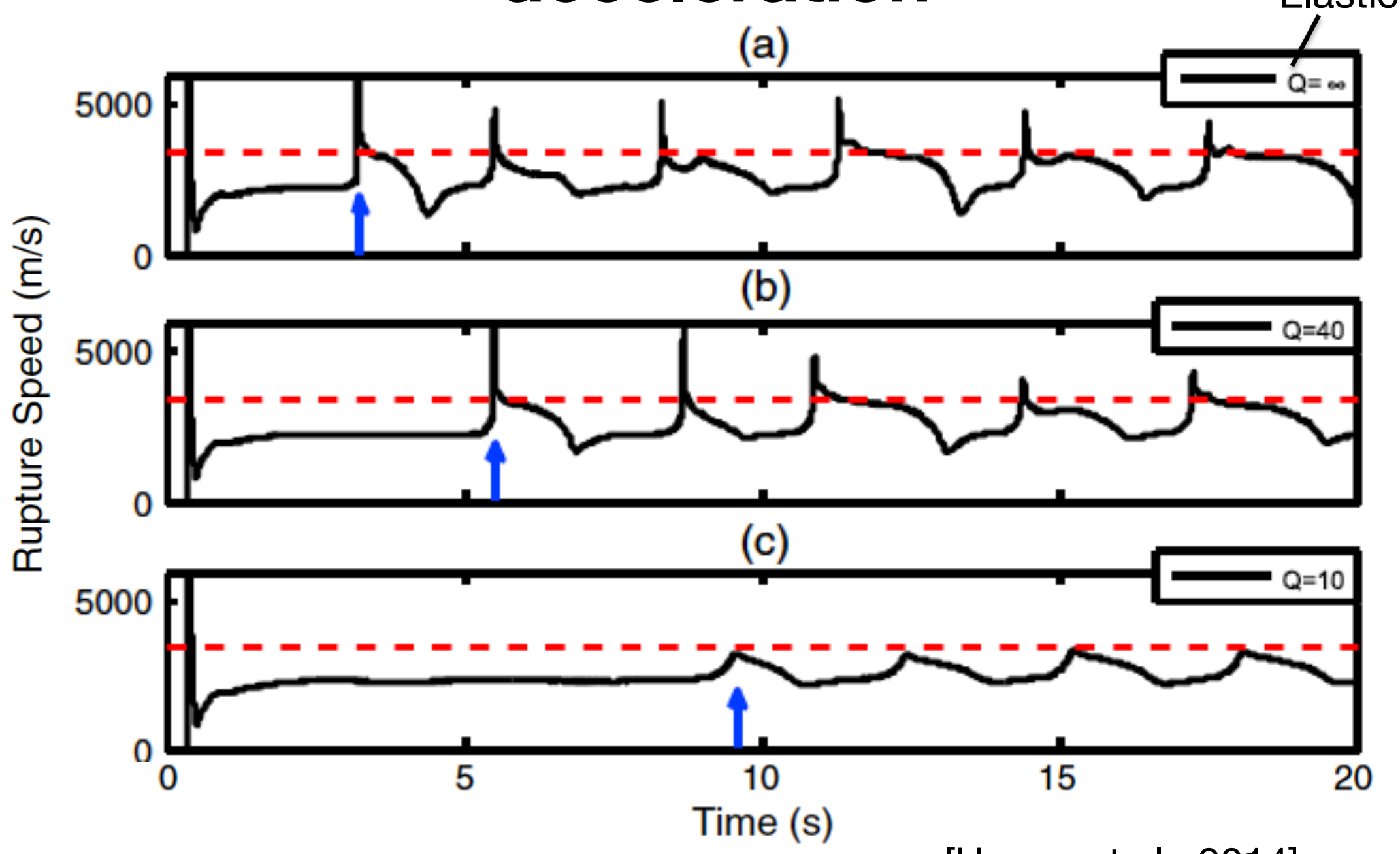

[Huang et al., 2014] 


\section{Earthquakes cause DFZ damage on both compressional and extensional sides}

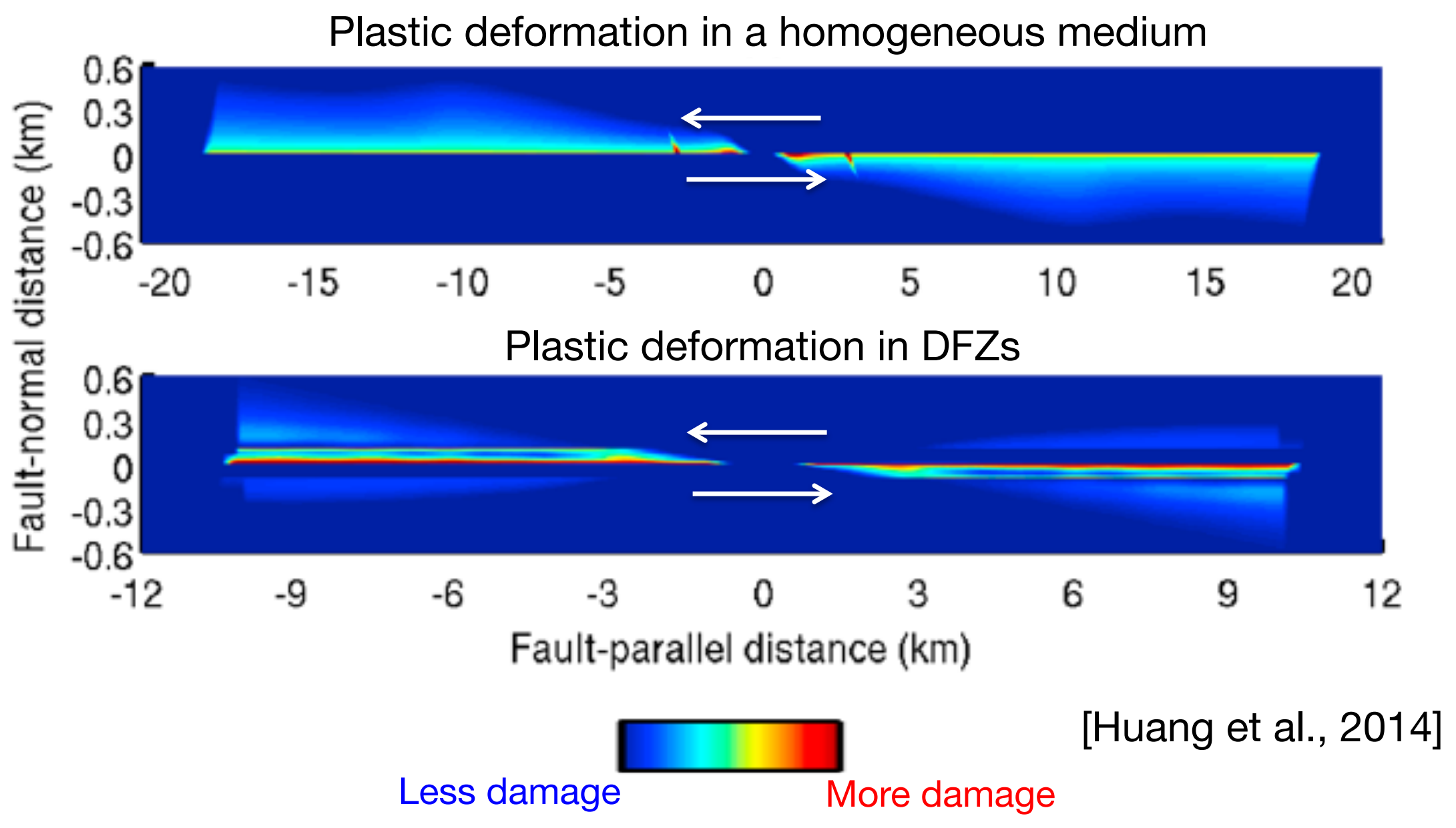




\section{DFZ damage preserves rupture pattern of previous earthquakes}
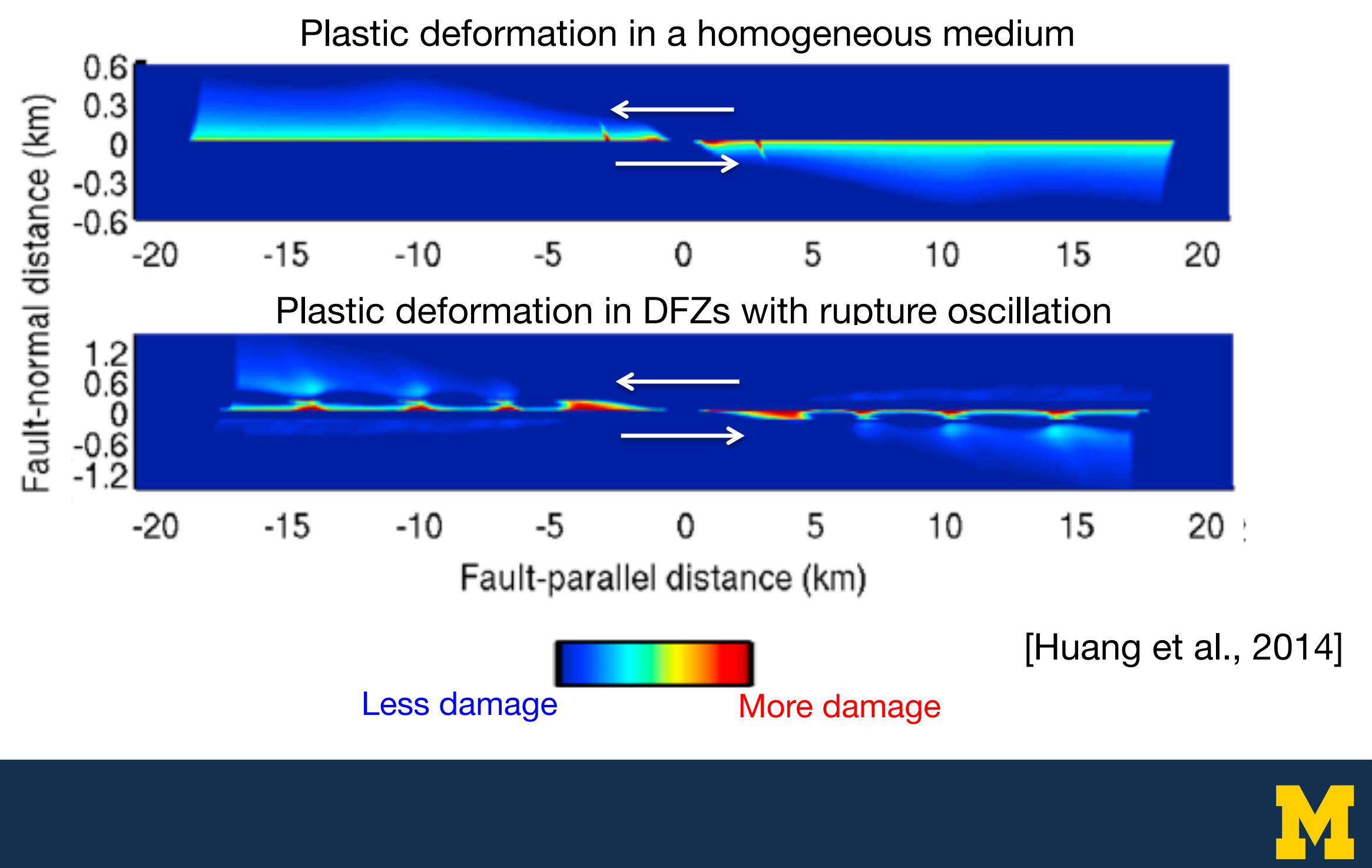


\section{Earthquake cycle models are needed to understand DFZ development}
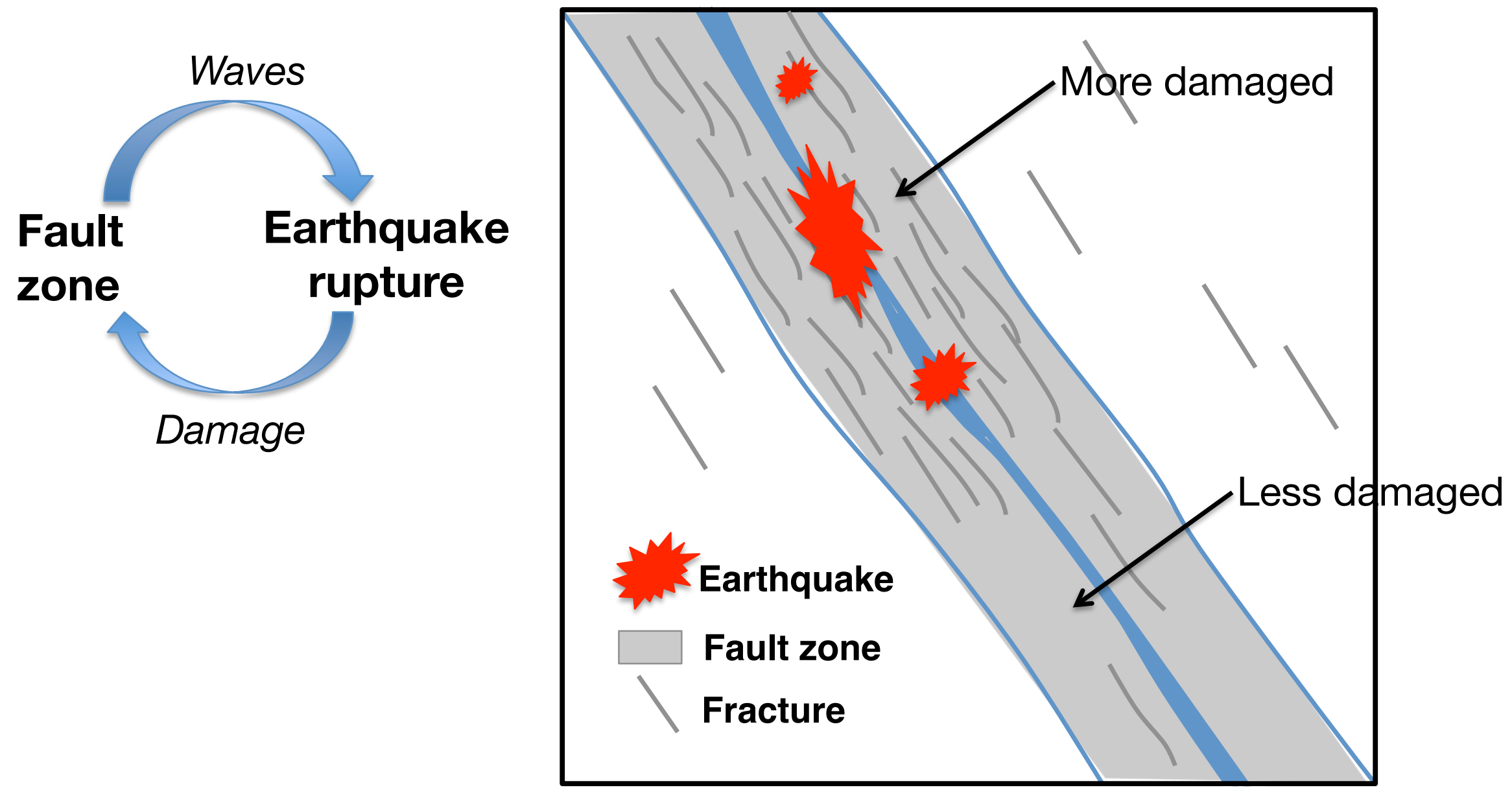